\title{
Preliminary characterization of a laser-generated plasma sheet
}

\author{
P. A. Keiter \\ University of Michigan, Ann Arbor, MI 48105 \\ G. Malamud \\ University of Michigan, Ann Arbor, MI 48105 \\ Department of Physics, Nuclear Research Center-Negev, Beer-Sheva, Israel
}

M. Trantham, J. Fein, J. Davis, S. R. Klein, R. P. Drake

University of Michigan, Ann Arbor, MI 48105

\begin{abstract}
We present the results from recent experiments to create a flowing plasma sheet. Two groups of three laser beams with nominally $1.5 \mathrm{~kJ}$ of energy per group were focused to separate pointing locations, driving a shock into a wedge target. As the shock breaks out of the wedge, the plasma is focused on center, creating a sheet of plasma. Measurements at $60 \mathrm{~ns}$ indicate the plasma sheet has propagated 2825 microns with an average velocity of 49 microns/ns. These experiments follow previous experiments [Krauland et al 2013], which are aimed at studying similar physics as that found in the hot spot region of cataclysmic variables. Krauland et al created a flowing plasma, which represents the flowing plasma from the secondary star. This flow interacted with a stationary object, which represented the disk around the white dwarf. A reverse shock is a shock formed when a freely expanding plasma encounters an obstacle. Reverse shocks can be generated by a blast wave propagating through a medium. They can also be found in binary star systems where the flowing gas from a companion star interacts with the accretion disk of the primary star.
\end{abstract}

Keywords: cataclysmic variable star, plasma flow, laboratory astrophysics

Email address: pkeiter@umich.edu (P. A. Keiter) 


\section{Introduction}

The night sky is filled with many points of light. Although these points of light appear as single stars, the majority of them are systems composed of multiple stars, most of which are binary star systems. Cataclysmic variables are a specific type of binary star system consisting of a low-mass main sequence secondary star that accretes mass to a white dwarf primary [1,2]. This accretion leads to bursts of light and hence variability in the light curves of these systems. Classification of cataclysmic variables depends on the light curve morphology. They include classical novae, dwarf novae, recurrent novae, nova-like variables and magnetic cataclysmic variables.

In most binary star systems, the stars are separated such that there is little to no gravitation interaction between them beyond their mutual attraction. However, if the stars are close, then tidal effects can become important. For a binary star system one can determine the Roche potentials and the Lagrangian points, which are locations where the force is zero. When one star fills its Roche lobe, the system is considered to be semi-detached. In general, most cataclysmic variables fall into this category. In these systems matter flows over the L1 Langranian point, which is located directly between the two stars, and then streams toward the white dwarf star and forms an accretion disk. Once the accretion disk is fully formed, the stream of material impacts into the outer rim of the accretion disk at supersonic speeds. This may create a shock-heated area which may radiate as much if not more energy at optical wavelengths than all the other components combined [1].

This area is referred to as the hot spot. As the stream impacts the disk, the kinetic energy of the stream is converted to radiation. The details of the interaction of the stream of gas and the accretion disk are not well known [3]. While it is well understood that a shock is formed, there is much uncertainty in the dynamics of the collision region. The dynamics of the interaction region depends on the radiative cooling properties of the system. Wu et al. [4] examined the accretion shock structure for the case where the cooling was primarily due to optically thick bremsstrahlung emission. Armitage and Livio [5] performed three dimensional simulations to examine the role of radiative cooling.

Laboratory experiments can be performed in order to better understand how radiative cooling can alter the dynamics of a shock. Previous laboratory experiments had demonstrated the creation of radiative shocks $[6,7]$, where the structure of the density and temperature is affected by the radiation from the shock-heated material. More recent work $[8,9]$ created the first laboratory reverse radiative shock.

Figure 1 compares the cataclysmic variable system and the experiment described in Krauland et al. [8]. Those experiments created a flowing plasma, which represents the stream of material from the secondary star in the cataclysmic variable. In those experiments, the flow interacted with a stationary object, which represents the disk around the white dwarf. When a suitable material was used to create the flow in those experiments, a reverse radiation shock was observed. Krauland et al. [9] characterized the reverse radiative shock and discussed the scaling between the laboratory and astrophysical parameters. The primary goal of these experiments was to understand the morphology of a strongly radiating collision region in the laboratory. Experiments have also been performed to study the physics that occurs in the create of accretion shocks 
in magnetized cataclysmic variables [10].

Since the accretion disk in the cataclysmic variable system is rotating, a next progression for the experimental platform is to replace the stationary object with dense flowing matter. There are two requirements for the moving object in order to make a connection to the cataclysmic variable system.

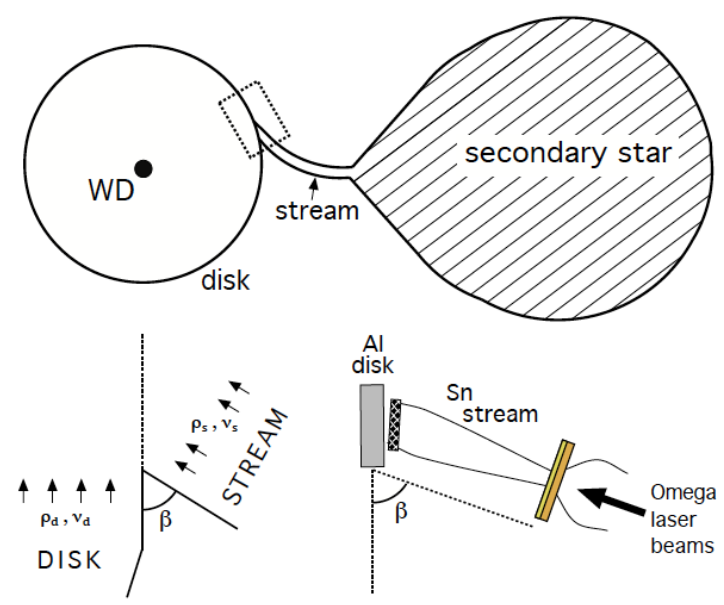

Figure 1: A schematic depicting the flow similarities between the cataclysmic variable system and the experiment described by Krauland et al. [8]. The figure is adapted from Krauland et al. [8].

First, we want the moving object to have a much longer length compared to the width. This is analogous to the accretion disk which has a diameter much greater than its thickness. Also, the density of the accretion disk is roughly 50 times greater than the stream of plasma from the secondary star. In order to meet these requirements we created a sheet of dense plasma.

The experiment described in this paper the creation of a plasma sheet. We will discuss the experimental configuration in Section 2, relevant simulations in Section 3, the results in Section 4.

\section{Experimental configuration}

The experiments were performed at the OMEGA laser facility [11]. A schematic of the wedge target is shown in Figure 2. It is composed of a plastic wedge and 15 micron thick $\mathrm{Cu}$ foil placed on the wedge surface. The $\mathrm{Cu}$ layer has multiple purposes. Simulations indicate the $\mathrm{Cu}$ is required to obtain the correct density for the plasma sheet. It also adds opacity to the plasma sheet, making it easier to image with x-ray radiography. The plastic wedge was 368 microns at its thickest, at the edges and 100 microns thick in the middle, so that the angle $\theta$ was 75 degrees.

Six laser beams were used to irradiate the target. Each laser beam used SG4 phase plates, producing a supergaussian profile and nominally had $500 \mathrm{~J}$ of energy. The six 


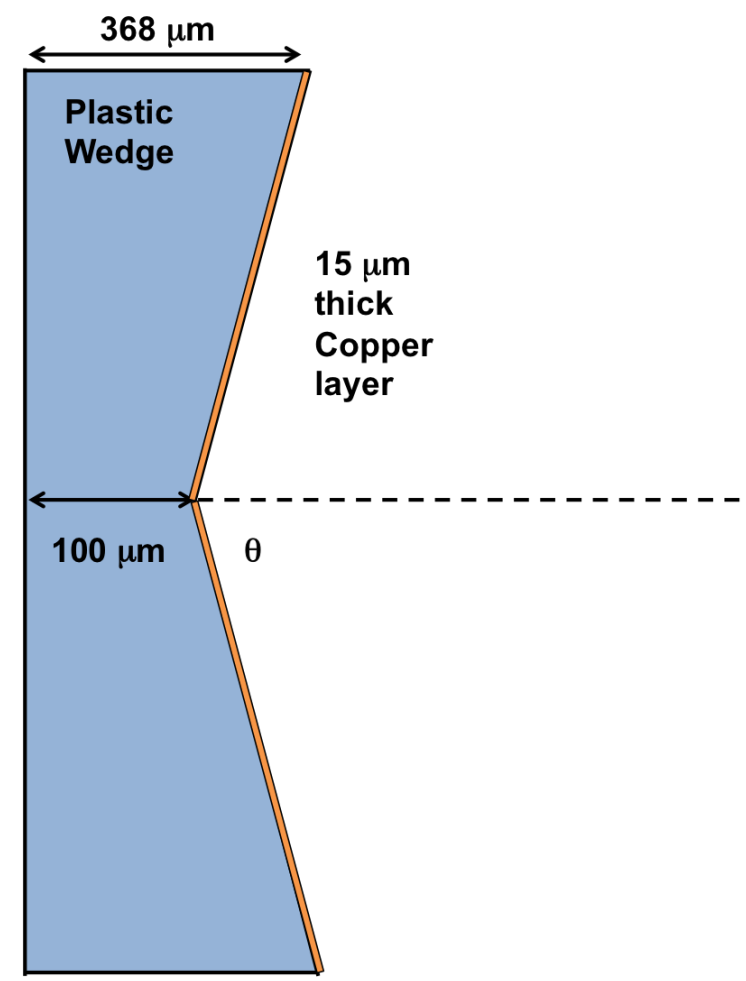

Figure 2: A schematic of the plasma sheet target. The wedge surface is angled to help direct material toward the target axis as the shock breaks out.

beams were distributed into two groups of three laser beams each. The centers of the laser beams were offset by 700 microns from each other along the direction of the wedge. Using the Visrad software, we could predict the laser irradiance on the plasma sheet target, which is shown in Figure 3. The black dashed line indicates where the trough of the wedge target is relative to the laser beams.

The lasers drive a shock wave in the plastic. The shock propagates through the plastic, breaking out at the center first. The shock continues to break out progressively further from the center of the wedge. Due to the shape of the wedge, the material is directed toward the axis, contributing to the plasma sheet.

The experimental configuration is shown in Figure 4. Dual-axis, orthogonal, x-ray radiography was used to diagnose the plasma sheet. The data were recorded on Agfa D8 film. The large acrylic cone and the gold shielding help shield the diagnostics from the ablated plasma from the drive surface. The backlighters used a pinhole apertured geometry with a tapered pinhole and a Vanadium (V) foil as the x-ray source. The pinhole is tapered from 50 microns closest to the $\mathrm{V}$ foil to 20 microns closest to the target. Radiographs were obtained using the $\mathrm{V} \mathrm{He}$-alpha line at $5.2 \mathrm{keV}$. A set of five polystyrene steps wedge was used a fiducial in order to infer an areal density from the measured transmission. The steps were nominally 50, 100, 250, 600 and 1200 microns 


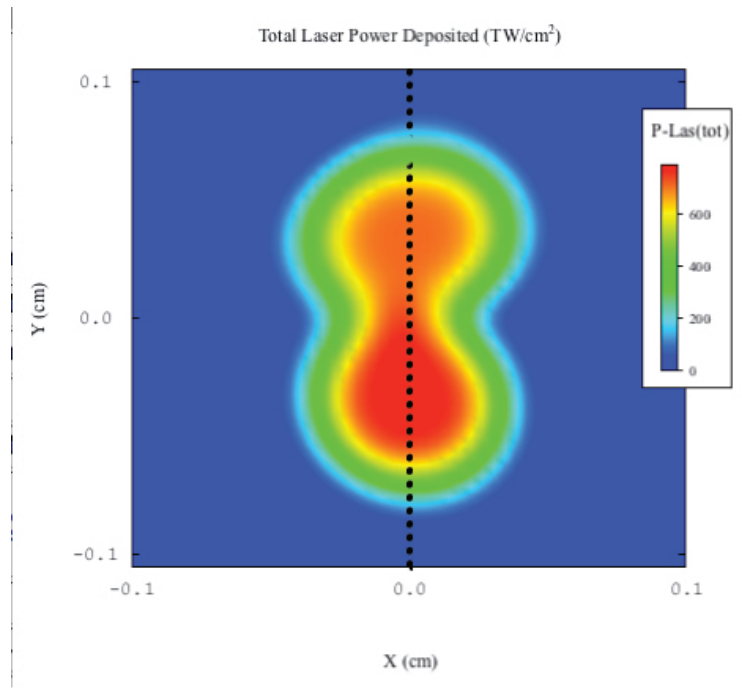

Figure 3: The predicted laser irradiation for 2 pointing locations with 3 laser beams pointing at each location. The black dashed line indicates where the trough of the wedge target is relative to the laser beams.

thick and had a density of $1.05 \mathrm{~g} / \mathrm{cm}^{3}$.

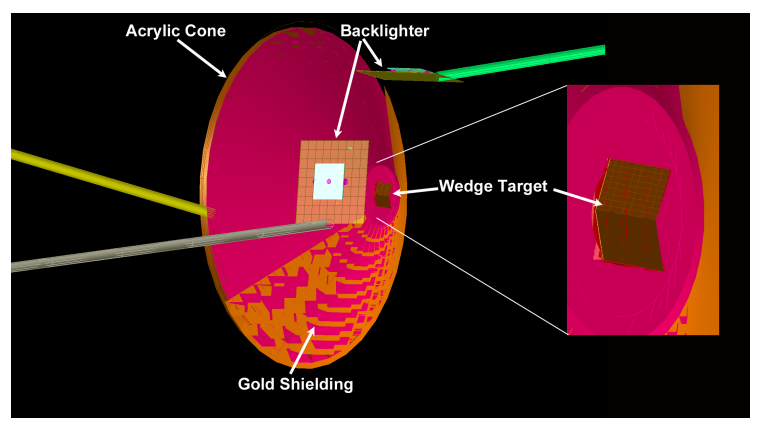

Figure 4: A schematic of the experimental configuration. The large acrylic cones shields the diagnostics from unwanted x-rays. A blow up of the wedge is shown.

\section{Simulations}

The experiment was simulated with the CRASH code, which is a block-adaptivemesh, radiation hydrodynamic solver capable of modeling high-energy-density systems. The CRASH code is a finite-volume code that uses an implicit method for solving electron heat conduction and multi-group radiation diffusion [12]. These simulations use thirty logarithmically separated photon energy groups with a lower bound of 0.1 $\mathrm{eV}$ and an upper bound of $20 \mathrm{keV}$. In addition, CRASH has a laser deposition package 
and these simulations used 3D ray tracing for the laser deposition. The simulations used four levels of AMR and had an effective resolution of 1.5 microns.

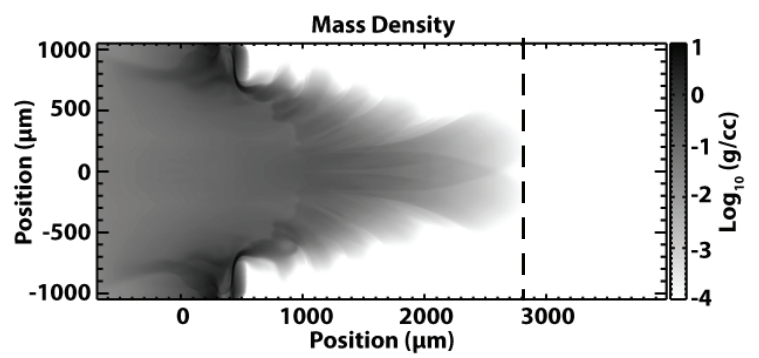

Figure 5: A plot of the simulated mass density of the plasma sheet at $60 \mathrm{~ns}$. The lasers are incident from the left and the plasma is flowing to the right. The dashed line is present to aid in the visualization of the tip of the plasma sheet.

We performed two-dimensional (2D) simulations in advance of the experiment, with results shown in Figure 5. In this geometry, the direction in and out of the page is considered infinite in dimension. This has the consequence of not capturing the finite laser spot size in that direction. The laser is incident from the left and the plasma sheet is propagating to the right. The tip of the plasma sheet has propagated 2825 microns. The density of the plasma sheet along the centerline ranges from roughly $10^{-2} \mathrm{~g} / \mathrm{cm}^{3}$ near the tip of the plasma sheet to $10^{-1} \mathrm{~g} / \mathrm{cm}^{3}$ near the base of the plasma sheet. The plasma sheet is about 800 microns in vertical extent near the tip.

\section{Experimental Results}

Figure 6 shows an experimental radiograph of the experiment at $60 \mathrm{~ns}$. In this image, the laser is incident from the left and the plasma sheet is propagating from left to right. The surface of the wedge target where the lasers irradiated it, is defined as $\mathrm{x}$ $=0$. One notices in Figure 6 that there are two regions of plasma, the larger region is roughly centered around $y=0$ and will be referred to as the plasma sheet. The smaller region and is roughly center at $\mathrm{y}=300$ microns and will be referred to as a secondary lobe. This secondary lobe will be discussed in more detail in the upcoming sections. Using the step wedge as spatial fiducial, an absolute spatial scale can be determined. Vanadium He-alpha x-rays at $5.2 \mathrm{keV}$ are used for the radiography.

Figure 7 shows contours of transmission, ranging from 0 - 1 with contours every 0.1 Using the 90 and $100 \%$ transmission contours, the position of the plasma sheet tip is located at $2875 \pm 25$ microns. This is only 50 microns (or 1.8\%) further than the simulations predicted. We can determine an average velocity of the plasma sheet by taking into account the thickness of the target (100 microns) and the time for the plasma sheet to break out of the target. We estimate this time to be roughly $4 \mathrm{~ns}$ from the simulations. Therefore the plasma sheet has an average velocity of roughly 49 microns/ns. 


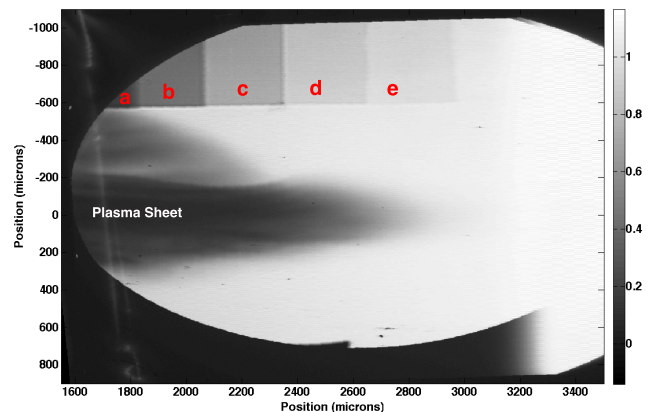

Figure 6: Side-on radiographic view of the plasma sheet at $60 \mathrm{~ns}$. The lasers are incident from the left and the plasma sheet is propagating to the right. A plastic step wedge provides acts as both a spatial and transmission fiducial. The labels on the step wedge correspond to the following thicknesses: a) 1200, b) 600, c) 250, d) 100 and e) 50 microns thick.

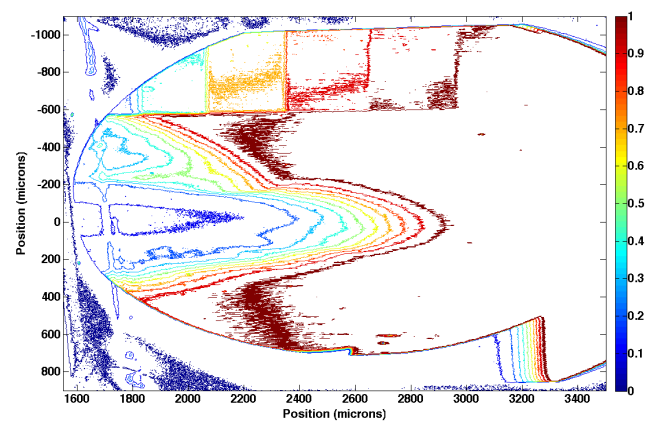

Figure 7: A contour plot the x-ray radiography from Figure 6. The contours represent transmission values ranging from 0 to 1 , with contours every 0.1 (or every $10 \%$ ).

\subsection{Determination of Plasma Sheet Density}

In order to determine the areal density of the plasma sheet along the diagnostic line of sight, the polystyrene step wedge is used as a fiducial. Static radiographs were obtained of one of the unshot plastic steps with a mono energetic x-ray source [13] using the Cr K-alpha line at $5414 \mathrm{eV}$. These radiographs confirm the areal density of the step wedge in the absence of high-energy background, such as is present in many high-energy density experiments. From the transmission through each of the steps and an independent measure of the thickness of each step we find the measured density agrees to within $10 \%$ of the accepted value of $1.05 \mathrm{~g} / \mathrm{cm}^{3}$ for polystyrene.

Determining an absolute density of the plasma sheet is complicated as the plasma sheet is composed of both plastic and copper. In addition, the width of the plasma sheet along the diagnostic axis is not well known. We are able to estimate an equivalent areal density of the plasma sheet, as follows. Figure 8 displays the transmission of the polystyrene step wedge (red curve) and the plasma sheet (black curve). 
The areal density measurements of the step wedge agree to within $10 \%$ of the areal density determined on the monochromatic x-ray source. By comparing the transmission of the plasma sheet to the transmission of the step wedge, we can determine that the plasma sheet, which includes the contributions from the plastic and copper, has a transmission equivalent to polystyrene with an areal density of $0.125 \mathrm{~g} / \mathrm{cm}^{2}$.

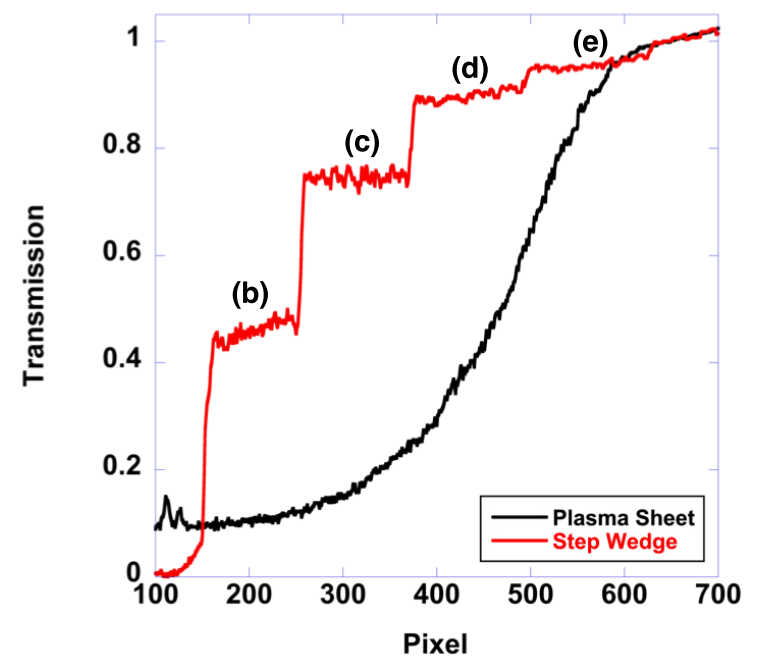

Figure 8: The transmission of the plasma sheet (black) and the polystyrene steps (red) along the horizontal direction. The labels on the steps (b-e) correspond to the steps labelled in Figure 6.

\subsection{Determination of Plasma Sheet Width}

We determined the width of the plasma sheet from vertical line outs of the experimental radiographs. The line out profiles were fit with a gaussian shape from which the full-width half-maximum (FWHM) of the profile was determined. Depending at which "x" value the vertical line out is obtained, the line out will intersect either the plasma sheet and the secondary lobe or just the plasma sheet. We obtained the limits of the plasma sheet width by employing two different methods. First we fit the data with a single Gaussian. This will overestimate the width of the plasma sheet where the secondary lobe is present. We then fit the data with a double Gaussian and obtained the width of the plasma sheet from the FWHM of the plasma sheet portion only. These results are plotted in Figure 9. The deduced width of the plasma sheet is less than 10\% of the distance the sheet has propagated.

The second lobe is likely caused by an offset of the lasers from the wedge centerline. One hypothesis is that a rotation error is responsible for the the misalignment. We have examined images taken of the targets by x-ray pinhole cameras and visible backlit images. We chose the outer edge of the plastic cone as a reference feature and compared the relative position on each shot. The difference in the position of the cone edge varied randomly from target to target. While the offset varied on each shot, the maximum offset from these images was roughly 290 microns. The cone diameter is 


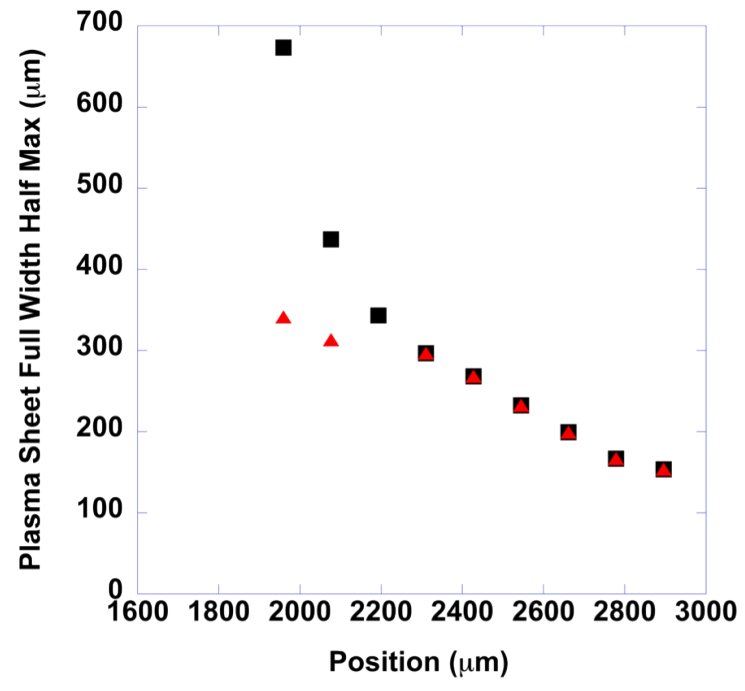

Figure 9: A plot of the measured plasma sheet width using a single gaussian (squares) and a double gaussian (triangles).

$28 \mathrm{~mm}$ and therefore a 290 micron difference in the positions would correspond to a rotational offset of 0.6 degrees. A beam offset would produce additional material on one side of the wedge, whose momentum would tend to deflect the main plasma sheet formed by the portion of the flow that is symmetric. Such a deflection seems to be present in Figure 6.

\subsection{Offset simulations}

In order to test the viability of the hypothesis that a systematic target offset created the secondary lobe, we performed additional simulations under the identical conditions as the base case, with the exception that the laser beams were offset 300 microns in the vertical direction. A synthetic radiograph of one of the simulations at $60 \mathrm{~ns}$ is shown in Figure 10. The formation of a secondary lobe can be observed in the simulations, giving plausibility to the theory that a secondary lobe can be formed if the laser beams are not pointing directly at the wedge center.

Differences exist between the offset simulation and the data (Figure 6) that we attribute to slightly different geometries between the simulation and experiment. In the simulations the lasers were offset only in the vertical direction. In the experiment, the target is constrained to rotate around the stalk and therefore an misalignment due to rotation will be a compound angle. In order to take this into account, threedimensional simulations would have to be performed. The CRASH code can perform three-dimensional simulations. 


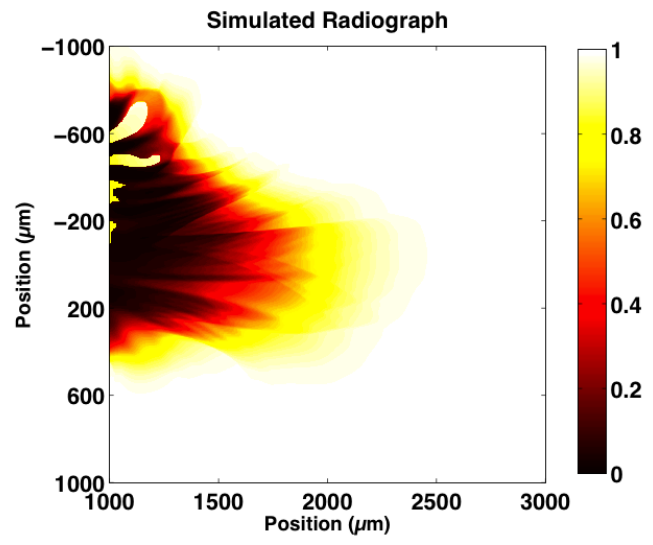

Figure 10: A synthetic radiograph of the plasma sheet experiment with the lasers offset 300 microns from the nominal location. The overall shape and formation of the secondary lobe indicates that laser beams offset from their nominal pointing location can create a secondary lobe.

\section{Conclusions}

We successfully produced a plasma sheet and determined the spatial extent of the plasma sheet from x-ray radiographs using the $5.2 \mathrm{keV}$ Vanadium He-alpha line. From at the radiography at $60 \mathrm{~ns}$, we inferred the average velocity of the plasma sheet was 50 microns/ns and the plasma width was less than 300 microns, which is less than $10 \%$ of the length of the plasma sheet. The plasma sheet width was found as a function of axial position. The plasma sheet has a transmission equivalent to the transmission of polystyrene with an areal density of $0.125 \mathrm{~g} / \mathrm{cm}^{2}$. The current estimates of the plasma sheet density indicate that it is roughly 50 times as dense as the plasma stream, meeting the scaling requirement to make it relevant to the disk-stream interactions of cataclysmic variables. The radiographs showed a secondary lobe, which is theorized to be due to a target misalignment of $<0.6$ degrees. Simulations with offset laser beams qualitatively reproduce the secondary lobe. Future target designs will improve the target alignment to eliminate the secondary lobe.

\section{Acknowledgements}

We would like to thank the OMEGA operations staff for their invaluable support. We would also like to thank Joe Cowan of Los Alamos National Lab and Fred Marshall of LLE for contributions. This work is funded by the U.S. Department of Energy, through the NNSA-DS and SC-OFES Joint Program in High-Energy-Density Laboratory Plasmas, grant number DE-NA0001840, and the National Laser User Facility Program, grant number DE-NA0000850, and through the Laboratory for Laser Energetics, University of Rochester by the NNSA/OICF under Cooperative Agreement No. DE-FC52-08NA28302. 


\section{References}

[1] B. Warner, Cataclysmic Variable Stars, Cambridge University Press, 1995.

[2] R. C. Smith, Contemporary Physics 47 (2006) 363-386.

[3] C. Hellier, Cataclysmic Variable Stars, Springer, 2001.

[4] K. Wu, G. Chanmugam, G. Shaviv, The Astrophysical Journal 426 (1994) 664668.

[5] P. J. Armitage, M. Livio, The Astrophysical Journal 493 (1998) 898.

[6] S. Bouquet, C. Stehle, M. Koenig, J.-P. Chieze, A. Benuzzi-Mounaix, D. Batani, S. Leygnac, X. Fleury, H. Merdji, C. Michaut, F. Thais, N. Grandjouan, T. Hall, E. Henry, V. Malka, J.-P. J. Lafon, Phys. Rev. Lett. 92 (2004) 225001.

[7] A. B.Reighard, R. P. Drake, K. K. Dannenberg, D. J. Kremer, M. Grosskopf, E. C. Harding, D. R. Leibrandt, S. G. Glendinning, T. S. Perry, B. A. Remington, J. Greenough, J. Knauer, T. Boehly, S. Bouquet, L. Boireau, M. Koenig, T. Vinci, Phys. Plasmas 13 (2006) 082901.

[8] C. M. Krauland, R. P. Drake, C. C. Kuranz, B. Loupias, T. Plewa, C. M. Huntington, D. N. Kaczala, S. Klein, R. Sweeney, R. P. Young, E. Falize, B. Villette, P. A. Keiter, Astrophysical Letters 762:L2 (2013).

[9] C. M. Krauland, R. P. Drake, C. C. Kuranz, R. Sweeney, M. Grosskopf, S. Klein, R. Gillespie, P. A. Keiter, B. Loupias, E. Felize, Phys. Plasmas 20 (2013) 056502.

[10] E. Falize, A. Ravasio, B. Loupias, A. Dizière, C. Gregory, C. Michaut, C. Busschaert, C. Cavet, M. Koenig, High Energy Density Physics 8 (2012) 1-4.

[11] J. M. Soures, R. L. McCrory, C. P. Verdon, A. Babushkin, R. E. Bahr, T. R. Boehly, R. Boni, D. K. Bradley, D. L. Brown, R. S. Craxton, J. A. Delettrez, W. R. Donaldson, R. Epstein, P. A. Jaanimagi, S. D. Jacobs, K. Kearney, R. L. Keck, J. H. Kelly, T. J. Kessler, R. L. Kremens, J. P. Knauer, S. A. Kumpan, S. A. Letzring, D. J. Lonobile, S. J. Loucks, L. D. Lund, F. J. Marshall, P. W. McKenty, D. D. Meyerhofer, S. F. B. Morse, A. Okishev, S. Papernov, G. Pien, W. Seka, R. Short, M. J. S. III, M. Skeldon, S. Skupsky, A. W. Schmid, D. J. Smith, S. Swales, M. Wittman, B. Yaakobi, Phys. Plasmas 3 (1996) 2108.

[12] B. van der Holst, G. Toth, I. V. Sokolov, K. G. Powell, J. P. Holloway, E. S. Myra, Q. Stout, M. L. Adams, J. E. Morel, S. Karni, B. Fryxell, R. P. Drake, The Astrophysical Journal Supplement Series. 194 (2011) 23.

[13] N. E. Lanier, C. Hamilton, J. M. Taccetti, Rev. Sci. Instrum. 83 (2012) 10E521. 